\title{
Evaluation of the Burkard Cyclonic Spore Sampler for Collection Efficiency of Ascospores
}

Carrie Larson, Leonard J. Francl, and Timothy Friesen, Department of Plant Pathology, North Dakota State University, Fargo, ND 58105

\begin{abstract}
Larson, C., Francl, L. J., and Friesen, T. 2001. Evaluation of the Burkard cyclonic spore sampler for collection efficiency of ascospores. Plant Dis. 85:1249-1252.

The commercially available Burkard cyclonic sampler takes in air volumetrically, creates a vortex in an aluminum cylinder, and deposits particulates in an Eppendorf vial. Retention within the cylinder was determined for ascospores released by cultures of Sordaria fimicola and Gibberella zeae. Manufacturer's recommended amperage level and reduced power were tested, and a surfactant was applied to the cylinder wall. Under recommended power, an average of $78 \%$ of the $S$. fimicola ascospores were collected in the vial, while $22 \%$ lodged inside the cylinder. Conversely, only $25 \%$ of the G. zeae ascospores were collected in the vial, while $75 \%$ remained lodged inside the cylinder. Application of a surfactant to reduce the adherence of ascospores on the cylinder wall, instead resulted in $83 \%$ of the S. fimicola ascospores and $99.7 \%$ of the $G$. zeae ascospores deposited on the cylinder wall. When the power supply was decreased from $200 \mathrm{~mA}$ to $140 \mathrm{~mA}$, the ratio of ascospores of $G$. zeae retained by the sampler remained nearly the same, indicating that the retention error was not a function of airflow rate within the tested power range. However, the total number of ascospores collected was significantly less under reduced power. A $90-\mathrm{A} / \mathrm{h}(12 \mathrm{~V})$ battery supplied greater than $150 \mathrm{~mA}$ when connected to the Burkard for 4 days at temperatures between 22 and $28^{\circ} \mathrm{C}$ and could maintain current above 190 $\mathrm{mA}$ for 7 days with a $21-\mathrm{W}$ solar panel. A $21-\mathrm{W}$ solar panel charging a $90-\mathrm{A} / \mathrm{h}$ battery should maintain the amperage needed for the Burkard to maintain proper flow rate in most environments. The aluminum cylinder should be rinsed out thoroughly when collecting samples of fungal ascospores or any other particulates with the propensity to adhere to the cylinder wall.
\end{abstract}

Additional keywords: aerobiology, disease forecasting

The science of aerobiology, which encompasses movement of plant pathogens and other microorganisms through the atmosphere, is integral to our understanding of pathogen dispersal mechanisms and is a valuable tool for disease forecasting (2). Many samplers have been devised to quantify microorganisms present in the air, either through active or passive means (6). Active samplers forcibly draw in air, while passive samplers rely on gravity or impaction to collect microorganisms (2). Much of the plant pathology literature on aerial spore dispersal is based on spore samples collected by the Burkard 7-day volumetric sampler and its progenitor, the Hirst 24-h sampler $(2,5)$.

In 1991, Burkard Manufacturing (Ricksmansworth, UK) introduced the Burkard cyclonic sampler, which draws in air at 16.5 liters/min, creates a vortex in an aluminum cylinder by means of a channel at the entrance, and deposits particulates from the air in a 1.5-ml Eppendorf vial. Advan-

Corresponding author: Leonard J. Francl E-mail: Leonard.Francl@ndsu.nodak.edu

Accepted for publication 14 August 2001.

Publication no. D-2001-1004-01R

(C) 2001 The American Phytopathological Society tages of the Burkard cyclonic sampler over the Burkard 7-day tape model include: virtually no risk of overloading the sampler, easier collection and transport of particles in an Eppendorf tube, slightly less expensive equipment, and less laborintensive sample counts, especially if using biochemical techniques such as a polymerase chain reaction (PCR) or immunological assays.

The Burkard cyclonic spore sampler has been tested by Burkard Manufacturing with pollen, carbon particles, and radioactive particles when operated under constant power (i.e., regulated alternating electric current). If the Burkard cyclonic sampler is operating on a battery that is discharging, the sampler may not maintain its specified flow rate. Also, fungal spores have a number of physical characteristics that make them different from pollen and inert particulates. Therefore, sampler performance was determined for ascospores from two fungal species, and the power curve was assessed when using a battery or battery plus solar panel.

\section{MATERIALS AND METHODS}

Efficiency of Burkard spore sampling. Two Ascomycete fungal species were chosen to determine performance of the Burkard cyclonic sampler. Sordaria fimicola was chosen because of the ease of inducing reproduction in culture, visibility of the dark ascospores, and long dispersal trajectory of the ascospores upon liberation (11). Gibberella zeae was chosen as the second species because of the ongoing research investigating the epidemiology of Fusarium head blight of wheat $(4,8)$.

To produce perithecia, cultures of $S$. fimicola were grown on half-strength potato dextrose agar (PDA) at $24 \pm 3^{\circ} \mathrm{C}$ under one UV light (F40/350B1, Sylvania, Danvers, MA) and three fluorescent lights (F40/CWX, Sylvania) for approximately 20 days. Sporulating cultures were transferred to a greenhouse room in which three Burkard cyclonic samplers were located. Each sampler was powered by a regulated $12-\mathrm{V}$ (3 A) power supply (model 22-504, RadioShack, Ft. Worth, TX) to maintain a constant voltage and a constant air intake of 16.5 liters/min. One plate of S. fimicola was placed in the bottom of a 1.36-liter aluminum can that had an opening for the orifice of the Burkard sampler cut at a height and width of $2 \times 11 \mathrm{~mm}$. The can was fit against the cylinder orifice to minimize leaking of air into the sampler from the outside. A hole was drilled in the can opposite the orifice hole to allow airflow through the can. A 1-cm-thick piece of clear Plexiglas was placed on top of the can to prevent air leaking into the sampler other than air drawn through the hole in the can. The system was left in this manner for $4 \mathrm{~h}$ to allow the ascospores of S. fimicola to discharge from the plate. Eppendorf vial samples were processed by adding $1 \mathrm{ml}$ of distilled water followed by a triple rinse of $1 \mathrm{ml}$ of distilled water per each rinse. Tween 20 was added at $1 \mathrm{ml}$ per $100 \mathrm{ml}$ of distilled water during rinsing to minimize spore clumping. Five milliliters of distilled water with Tween was added to the inside of the cylinder, shaken for $30 \mathrm{~s}$, and the rinsate collected. Ascospores in three 5- $\mu$ l drops were counted, from which the mean number of ascospores was calculated for the vial and cylinder spore solutions. This experiment was performed 15 times.

An isolate of G. zeae, Hagar 94, was plated on half-strength PDA (3). Conidia of Fusarium graminearum were harvested from the plates in distilled water after 5 days and diluted to produce a suspension of 4,000 spores per $\mathrm{ml}$. Two hundred microliters of this spore suspension were plated onto carrot agar $(3,9)$ and incubated for 5 to 6 days at $24 \pm 3^{\circ} \mathrm{C}$ under one UV and three fluorescent lights to produce mycelium. Mycelium was flattened with a 
test tube and left to continue growth under the same conditions until perithecia formation and evidence of ascospore discharge of $G$. zeae could be seen on the lid of the petri dish under a stereomicroscope.

The Burkard cyclonic sampler was evaluated for ascospore collection of $G$. zeae using the same procedures as with $S$. fimicola, except for minor changes in the vacuum apparatus because initial trials showed too few spores were being collected. The can was replaced with a $10-\mathrm{cm}-$ diameter PVC pipe. A hole was drilled in one side for the cylinder orifice, and a second hole was drilled opposite for airflow. Plexiglas was glued to the bottom of the PVC pipe. Another piece of clear Plexiglas was placed on the top of the pipe to reduce air leakage. After $24 \mathrm{~h}$, samples were collected and counted microscopically. The experiment was performed 15 times using three samplers. Ascospores of $G$. zeae collected in the sampler were counted in the same manner as for S. fimicola, except the volume of distilled water used to rinse the Eppendorf vial and cylinder was either 10 or $20 \mathrm{ml}$, depending on the density of the spore concentration.

In an attempt to increase efficiency of the Burkard cyclonic spore sampler at collecting fungal spores, Sigmacote, an organopolysiloxane in heptane product (Sigma, St. Louis, MO), was applied to the aluminum cylinder by immersion in the liquid product each time a test was initiated. Sigmacote forms a tight, microscopi-

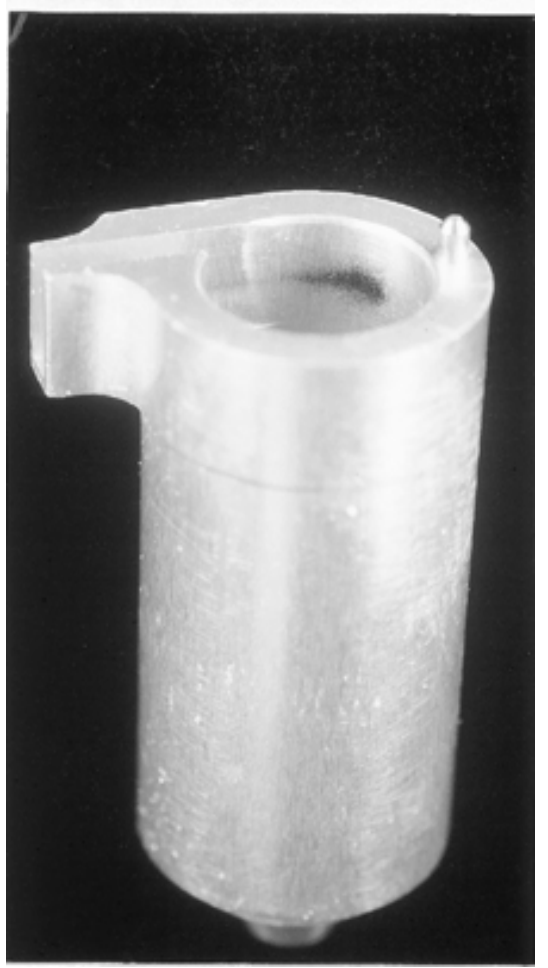

Fig. 1. Aluminum vortex chamber of the Burkard cyclonic spore sampler showing ascospores of Sordaria fimicola deposited on the inner wall near the external orifice. cally thin film of silicone on the surface to reduce adherence of biological molecules. The Burkard cyclonic sampler was tested with each of the two fungal species alone, and with Sigmacote applied to the collection cylinder. The spore collection and sampling procedure described above was used with Sigmacote applied to the cylinder. The experiment was performed six times with three replicate Burkard samplers.

Burkard power curve response. The regulated $12-\mathrm{V}$ power supply to the Burkard cyclonic sampler was decreased from $200 \mathrm{~mA}$ to $140 \mathrm{~mA}$, by means of an in-line resistor of $22 \mathrm{ohms}$, to simulate declining battery power. Spore counts of G. zeae were assessed in the same manner as described above. This experiment was performed six times while alternating two Burkard cyclonic samplers at full and decreased power.

Tests were also carried out to determined the flow of electricity to the Burkard cyclonic spore sampler when powered by a $90-\mathrm{A} / \mathrm{h}(12-\mathrm{V})$ lead cell battery. Readings of the current battery power level were taken at 10-min intervals for 9 days using a 24-range digital multimeter with a PC interface (model 22-805, RadioShack) wired in series with a laptop computer, battery, and Burkard cyclonic spore sampler. The data were recorded through the laptop computer where it could be downloaded daily. The greenhouse temperature during this experiment ranged from 20 to $28^{\circ} \mathrm{C}$. Another test was carried out to determine the current flow to a Burkard cyclonic sampler powered with a Unisolar 21-W solar collector equipped with a voltage regulator (Northern Arizona Wind \& Sun, Flagstaff, AZ). This experiment was run under similar greenhouse conditions for 7 days in December 1999 and August 2000.

\section{RESULTS}

After each experiment, ascospores of $S$. fimicola and G. zeae could be found on the inner walls of the aluminum cylinder (Fig. 1). Deposits of ascospores were most concentrated near the external orifice entrance of the sampler and in the vortex channel.

Retention error, defined as the proportion of spores not deposited in the collection vessel (i.e., Eppendorf vial) relative to the total number of spores collected in the air stream intake (2), was calculated for each trial. The results showed a serious inefficiency of the Burkard cyclonic sampler at collecting fungal spores (Table 1). Moreover, retention error increased when cylinder walls were covered with Sigmacote. G. zeae apparently was sampled less efficiently than $S$. fimicola under these conditions. However, results for the two species were not compared statistically because experiments were conducted at different times.

When the power supply used to operate the Burkard cyclonic sampler was de-

Table 1. Numbers of ascospores of two fungal species collected in the Eppendorf vial and the vortex cylinder of the Burkard cyclonic sampler, with and without a silicone coating on the cylinder (Sigmacote)

\begin{tabular}{lcccc}
\hline Fungal species & $\begin{array}{c}\text { Vial } \pm \\
\text { SD }^{\mathbf{w}}(\boldsymbol{\%})\end{array}$ & $\begin{array}{c}\text { Cylinder } \pm \\
\mathbf{S D}^{\mathbf{x}}(\boldsymbol{\%})\end{array}$ & $\begin{array}{c}\text { Mean vial } \\
\text { spore count }^{\mathbf{y}}\end{array}$ & $\begin{array}{c}\text { Mean cylinder } \\
\text { spore count }^{\mathbf{z}}\end{array}$ \\
\hline Sordaria fimicola & $76 \pm 3.2$ & $24 \pm 3.2$ & 118,320 & 38,236 \\
Gibberella zeae & $20 \pm 4$ & $80 \pm 4$ & 110,772 & 633,921 \\
S. fimicola + Sigmacote & $17 \pm 8.4$ & $83 \pm 8.4$ & 8,708 & 123,558 \\
G. zeae + Sigmacote & $0.3 \pm 0.2$ & $99.7 \pm 0.2$ & 55,054 & $1,116,333$ \\
\hline
\end{tabular}

${ }^{a}$ Percentage of ascospores in the Eppendorf vial \pm standard deviation (SD).

b Percentage of ascospores lodged in the vortex cylinder \pm standard deviation (SD).

${ }^{c}$ Average number of ascospores in the Eppendorf vial after 15 replications.

${ }^{d}$ Average number of ascospores in the vortex cylinder after 15 replications.

Table 2. Numbers of ascospores of Gibberella zeae collected in the Eppendorf vial and the vortex cylinder of the Burkard cyclonic sampler when operated with normal and decreased power

\begin{tabular}{lccccc}
\hline Fungal species & $\begin{array}{c}\text { Vial } \pm \\
\text { SD }^{\mathbf{t}}(\boldsymbol{\%})\end{array}$ & $\begin{array}{c}\text { Cylinder } \pm \\
\mathbf{S D}^{\mathbf{u}}(\boldsymbol{\%})\end{array}$ & $\begin{array}{c}\text { Average vial } \\
\text { spore count }^{\mathbf{v}}\end{array}$ & $\begin{array}{c}\text { Average cylinder } \\
\text { spore count }^{\mathbf{w}}\end{array}$ & $\begin{array}{c}\text { Total spore } \\
\text { count }^{\mathbf{x}}\end{array}$ \\
\hline $\begin{array}{l}\text { G. zeae } \\
200 \mathrm{~mA}^{\mathrm{y}}\end{array}$ & $17 \pm 2.9$ & $83 \pm 2.9$ & 182,333 & $1,019,000$ & $1,201,333 \mathrm{a}$ \\
$\begin{array}{l}\text { G. zeae } \\
140 \mathrm{~mA}^{\mathrm{z}}\end{array}$ & $13 \pm 1.9$ & $87 \pm 1.9$ & 64,520 & 687,200 & $751,720 \mathrm{~b}$ \\
\hline
\end{tabular}

${ }^{t}$ Percentage of ascospores in Eppendorf vial \pm standard deviation (SD).

" Percentage of ascospores in vortex cylinder \pm standard deviation (SD).

${ }^{v}$ Average number of ascospores in the Eppendorf vial after six replications.

${ }^{\mathrm{w}}$ Average number of ascospores in the vortex cylinder after six replications.

$\mathrm{x}$ Average number of total ascospores in the Eppendorf vial and in the vortex cylinder. Means were identical with a probability of less than $10 \%$ according to a $t$ test.

${ }^{y} G$. zeae ascospores tested for collection efficiency in the Burkard cyclonic sampler at normal power.

${ }^{\mathrm{z}} G$. zeae ascospores tested for collection efficiency in the Burkard cyclonic sampler at decreased power. 
creased from $200 \mathrm{~mA}$ to $140 \mathrm{~mA}$, the difference in spore ratios from vial to cylinder was insignificant, but a larger difference $(P$ $=0.09$ ) was found for average spore counts between normal and reduced power (Table 2). A Burkard cyclonic spore sampler powered by a $90-\mathrm{A} / \mathrm{h}$ battery maintained power above $150 \mathrm{~mA}$ for approximately 4 days (Fig. 2). When the battery for the Burkard cyclonic sampler was powered by a solar collector, amperage was maintained above $190 \mathrm{~mA}$ indefinitely in the summer (Fig. 3) and for short winter day lengths (data not shown).

\section{DISCUSSION}

The degree of ascospore deposition on the cylinder wall of the Burkard cyclonic sampler would cause underrepresentation of sample results. The collecting cylinder (Fig. 1) must be rinsed thoroughly to minimize retention error. A single rinse of the cylinder removed $98 \%$ of the G. zeae ascospores lodged inside.

The liquid silicone failed to prevent ascospores from sticking inside the collecting cylinder and, in fact, seemed to have the opposite effect. Although the reason for this is unknown, a hydrophobic and ionic electrostatic interaction may have caused the ascospores to adhere to the silicone coat. Sigmacote is a silicone solution in heptane that readily forms a covalent microscopically thin film on surfaces and is water repellent. Since most fungal spores have a negative charge, a positively charged substratum would explain the ionic attraction between the two components (7). Fungal spores have the ability to adhere to a number of different surfaces, including Teflon, polystyrene, glass, cellophane, nutrient and water agars, and plant leaves $(1,7)$. In the case of S. fimicola, a 3$\mu \mathrm{m}$-thick layer of mucilage around each spore plays a role in spore attachment to surfaces (6). Also, the release of fungal adhesive materials appears to be a prerequisite for spore adherence by pathogenic fungi (10).

The decreased power of the regulated power supplies decreased the numbers of ascospores sampled $(P<0.10)$ but did not have a large effect on deposition of spores on the wall of the sampler. Airflow of the sampler is dependent on the RPMs of an unregulated motor. This result suggests that the airflow rate does not affect retention errors when near recommended operating levels. However, calibration is important for maintenance of the recommended rate of air intake. This study was conducted using equipment calibrated at the factory with a hot-wire anemometer. We found that a flowmeter (Thermal Mass

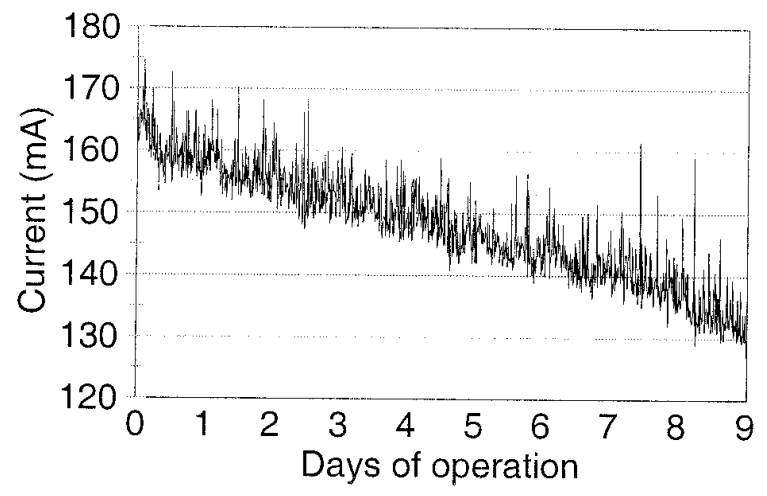

Fig. 2. Current draw of a Burkard cyclonic spore sampler operated with a $90-\mathrm{A} / \mathrm{h}$ battery in a greenhouse at $24 \pm 4^{\circ} \mathrm{C}$ in December 1999.

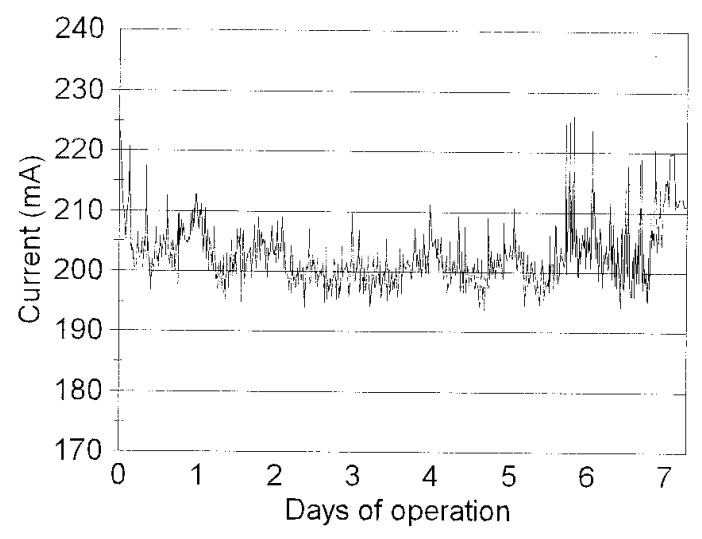

Fig. 3. Current flow of a Burkard cyclonic spore sampler operated with a $21-\mathrm{W}$ solar collector powering a $90-\mathrm{A} / \mathrm{h}$ battery. Tests were performed in a greenhouse at $24 \pm 4^{\circ} \mathrm{C}$ in August 2000 .

Flowmeter Model 4040, TSI, St. Paul, MN) gave a sensitive calibration response for the airflow rate of the Burkard cyclonic sampler.

The rate of decrease in amperage of the Burkard cyclonic sampler was more rapid that manufacturer guidelines would suggest. The $90-\mathrm{A} / \mathrm{h}$ batteries should have been able to power the Burkard for at least 7 days. However, after 4 days, the amperage dropped below $150 \mathrm{~mA}$, at which level the Burkard cyclonic sampler does not operate at 16.5 liters $/ \mathrm{min}$. In order to maintain this airflow rate, the Burkard cyclonic spore sampler must be operated at approximately $200 \mathrm{~mA}$.

A $90-A / h$ battery was kept charged by a 21-W solar panel at $47^{\circ} \mathrm{N}$ latitude near the time of winter solstice, when there were approximately $8.5 \mathrm{~h}$ of daylight (data not shown). Since our solar panel and battery gave a constant power curve, we expect a solar panel of $10 \mathrm{~W}$ or greater should be sufficient adjunct to a similarly sized battery to power the Burkard spore sampler above $200 \mathrm{~mA}$ during the growing season at a temperate latitude.

The Burkard cyclonic sampler was found to have a strong positive relationship with the Burkard 7-day volumetric tape model for pollen counts (G. Wili, personal communication) and can be recommended to plant pathologists and aerobiologists if methods are followed to alleviate serious shortcomings in retention of particles that have a tendency to adhere to the cylinder wall. A double or triple rinse should recover nearly all retained spores, and centrifugation of rinsate should allow for PCR or immunological analysis on a reduced volume.

This study involved only two ascosporeproducing species and freshly liberated ascospores. Different results may be obtained for other spore types and fungal species.

\section{LITERATURE CITED}

1. Braun, E. J., and Howard, R. J. 1994. Adhesion of Cochliobolus heterostrophus conidia and germlings to leaves and artificial surfaces. Exp. Mycol. 13:274-288.

2. Campbell, C. L., and Madden, L. V. 1990. Introduction to Plant Disease Epidemiology. Wiley Interscience, New York.

3. Dhingra, O. D., and Sinclair, J. B. 1985. Basic Plant Pathology Methods. 2nd ed. CRC Press, Boca Raton, FL.

4. Francl, L., Shaner, G., Bergstrom, G., Gilbert, J., Pedersen, W., Dill-Macky, R., Sweets, L., Corwin, B., Jin, Y., Gallenberg, D., and Wiersma, J. 1999. Daily inoculum levels of Gibberella zeae on wheat spikes. Plant Dis 83:662-666.

5. Hirst, J. M. 1952. An automatic volumetric spore trap. Ann. Appl. Biol. 39:257-265.

6. Ingold, C. T. 1971. Fungal Spores: Their Liberation and Dispersal. Oxford University Press, London.

7. KerChung, K., and Hoch, H. C. 1996. Germination of Phyllosticta ampelicida pycnidiospores: Prerequisite of adhesion to the substratum and the relationship of substratum wettability. Fungal Genet. Biol. 20:18-29. 
8. Markell, S., and Francl, L. J. 2001. Relationships among environmental variables, ascospore dispersal, and inoculum of Gibberella zeae. Phytopathology 91:S179.

9. Nelson, P. E., Toussoun, T. A., and Marasas,
W. F. O. 1983. Fusarium species. Pennsylvania State University Press, College Park.

10. Schuerger, A. C., and Mitchell, D. J. 1993 Influence of mucilage secreted by macroconidia of Fusarium solani f. sp. phaseoli on spore attachment to roots of Vigna radiata in hydroponic nutrient solution. Phytopathology 83:1162-1170.

11. Stevens, R. B., ed. 1974. Mycology Guidebook University of Washington Press, Seattle. 